\section{Mixed Phenotypic Acute Leukemia}

\author{
Sameer Kulkarni', Michael Lill² and Dimitrios Tzachanis ${ }^{2 *}$ \\ ${ }^{1}$ Department of Internal Medicine, Cedars-Sinai Medical Center, Los \\ Angeles, USA \\ ${ }^{2}$ Blood and Marrow Transplantation Program, Cedars-Sinai Medical \\ Center, Los Angeles, USA
}

\section{Introduction}

The majority of acute leukemias can be classified by the lineage of origin of blast cells as either myeloid, B Lymphoblastic (B-ALL) or T lymphoblastic. Acute leukemias are categorized by flow cytometry and now, increasingly, molecular results which help to determine whether the leukemia arises from the myeloid or lymphoid series. There does exist, however, an undefined or ambiguous group of leukemias in need of further characterization. Previous World Health Organization (WHO) criteria had defined a population of leukemic cells with no clear lineage restricting markers as Acute Undifferentiated Leukemias (AUL). Mixed Phenotypic Acute Leukemias (MPAL) represent a relatively new diagnostic designation, only being formally recognized and defined by the World Health Organization in 2008 [1]. Given the short time that these formal criteria have been established and the rarity of MPAL, there is a paucity of data regarding the precise biologic characterization of the disease as well as established guidelines on the management and prognosis of these patients. Herein, we review the defining characteristics, cytogenetic and clinical features and potential treatment options available for these patients.

\section{Defining Mixed Phenotypic Leukemias}

MPAL are quite rare, comprising roughly $3 \%$ of all new acute leukemia diagnoses. In order to establish the diagnosis, a specific set of pathologic criteria must be met to assign a designation of MPAL. Prior to 2008, mixed phenotypic leukemias were classified by a scoring system developed by the European Group for the Immunologic Classification of Leukemias (EGIL) (Table 1). The EGIL scoring system represented a refinement on a previous scoring system developed by Catovksy et al., in 1991 [2]. In this first scoring system, points were assigned to a limited number of Immune-Histochemical (IHC) markers from either a B cell, T cell or myeloid lineage. At the time, the Cavotsky scoring system weighted CD 22, CD3 and MPO

${ }^{*}$ Corresponding author: Dimitrios Tzachanis, Cedars-Sinai Medical Center, 8700 Beverly Blvd, AC1046-A Los Angeles, CA 90048, USA, Tel: 3104231279; Fax: 3109673819; E-mail: Dimitrios.Tzachanis@cshs.org

Citation: Kulkarni S, Lill M, Tzachanis D (2014) Mixed Phenotypic Acute Leukemia. J Hematol Blood Transfus Disord 1: 001.

Received: August 02, 2014; Accepted: October 25, 2014; Published: November 10, 2014 most heavily for defining their respective lineages. A score of greater than or equal to 2 was required to assign a lineage. If leukemia scored positively in more than one lineage, it was given the label of biphenotypic [2].

\begin{tabular}{|c|c|c|c|}
\hline Points & B Lineage & T Lineage & Myeloid Lineage \\
\hline 2 & CD 79a & CD3 (cyt/m) & Anti-MPO \\
\hline & cyt IgM & Anti-TCR alpha/beta & \\
\hline & cyt CD22 & Anti-TCR gamma/delta & \\
\hline 1 & CD 19 & CD 2 & CD 13 \\
\hline & CD 10 & CD 5 & CD 33 \\
\hline & CD 20 & CD 8 & CDw65 \\
\hline 0.5 & CD 10 & CD 117 \\
\hline & CD 25 & TdT 14 \\
\hline & & CD 7 $1 a$ & CD 15 \\
\hline
\end{tabular}

Table 1: EGIL Classification system.

Abbreviations: cyt: Cytogenetic; m: Monoclonal; MPO: Myeloperoxidase; TCR: T-Cell Receptor; Tdt: Terminal Deoxynucleotidyl Transferase

Note: $>2$ points required to assign a linage designation

The EGIL scoring system added new immune-histochemical markers to improve upon the accuracy of diagnosing ambiguous leukemias. For example, CD79a, a marker known to have strong specificity, was added to the $\mathrm{B}$ cell lineage [3]. To detect a positive antigen, $20 \%$ of cells had to express the IHC markers. According to the EGIL, only $10 \%$ of cells had to stain for CD3, MPO and CD79a, owing to their increased specificity. A score of greater than two was required to designate the presence of a particular lineage. A distinction under this system was continued in which leukemias were considered to be bilineal if they had two distinct blast populations each expressing one lineage or biphenotypic if one blast population co-expressed multiple lineage markers (Weinberg and Arber 2010). Both of these groups would often broadly be defined as leukemias of ambiguous origin. There remained drawbacks to this EGIL scoring system, though, that made accurate classification of these leukemias difficult. As an example, AML with $\mathrm{t}(8 ; 21)$ often expressed $\mathrm{B}$ cell markers including CD 19, CD 20 and CD 79a [4]. Depending on the number of $B$ cell surface markers present, these leukemias could garner anywhere from two to four points on the EGIL system, leading to a misclassified biphenotypic leukemia. Additionally, clearly defined B-cell acute leukemias often co-express MPO in up to $23 \%$ of cases [5].

The WHO reclassification in 2008 represents an effort to simplify and clarify the diagnosis of MPAL (Table 2). First, bilineal and biphenotypic leukemias have been brought together under one diagnosis. A clear delineation was made to exclude genetically defined lesions, such as $\mathrm{t}(15 ; 17), \mathrm{t}(8 ; 21)$ and $\operatorname{inv}(16)$. These core binding factors are AML defining and as such, the presence of B cell markers in these cases would not be classified as biphenotypic [1]. Additionally, irrespective of markers leukemias with complex karyotypes or other MDS related cytogenetics without previous evidence of MDS should all be classified as AML with myelodysplastic changes [1]. The most significant changes in the WHO criteria are related to very specific definitions for assigning a particular lineage. 


\begin{tabular}{|l|l|}
\hline Lineage & Markers \\
\hline Myeloid & $\begin{array}{l}\text { Myeloperoxidase } \\
\text { OR } \\
\text { Monocytic differentiation (at least two of the following: } \\
\text { NSE, CD11c, CD14, CD64, lysozyme) }\end{array}$ \\
\hline T lineage & $\begin{array}{l}\text { Cytoplasmic CD3 } \\
\text { OR } \\
\text { Surface CD3 }\end{array}$ \\
\hline B Lineage & $\begin{array}{l}\text { Strong CD 19 AND at least one of the following: } \\
\text { CD79a, cytoplasmic CD22 or CD 10 } \\
\text { OR } \\
\text { Weak CD19 AND at least two of the following: } \\
\text { CD79a, cytoplasmic CD22 or CD 10 }\end{array}$ \\
\hline
\end{tabular}

Table 2: 2008 WHO Classifications of leukemias of ambiguous lineage.

Myeloid defining markers include demonstration of immune-histochemial, flow cytometry or cytochemistry positivity of Myeloperoxidase (MPO) [4]. Additionally, presence of monocytic maturation is also evidence of myeloid differentiation. B cell lineage is defined by the expression of strong CD19 plus a secondary B-cell marker such as CD79a, cytoplasmic CD22 or CD10. Additionally, B cell lineage can also be defined by weak CD 19 marking with at least two of the three secondary B-cell markers. T cell lineage is defined by CD3 expression either in its cytoplasmic or surface form. A cohort of 100 patients who met the criteria for MPAL revealed that the $B$ cell/myeloid subtype of MPAL made up the majority of cases. 59 of the 100 patients had B cell/myeloid MPAL versus 35 who demonstrated a T-cell/myeloid subtype. The rest were either classified as T/B cell or trilineage populations $[4,6]$. As we shall discuss later, these changes have led to a change in the perceived incidence of these ambiguous leukemias.

\section{Clinical Features}

The majority of presenting symptoms in mixed phenotypic leukemias are not strikingly different from other forms of acute leukemia. The presence of bone marrow failure, as manifested by anemia and thrombocytopenia, is common. This disease affects patients in the pediatric population as well as adults. There is a slight male preponderance with a ratio of 1.6 to 1 in favor of men in a cohort of 100 patients [7]. Additionally, MPAL have been associated with rather high white counts at presentation [6]. The incidence of the disease has been difficult to pinpoint, mainly owing to the relatively new diagnostic category. In 2009, Al-Seraihy et al., [8] reported on a group of 633 new cases of acute leukemia. Of these, 24, or 3.8\%, were reported as being biphenotypic based on the EGIL criteria [8]. Of these, only roughly $1.7 \%$ would still be classified as MPAL under the WHO criteria. Owaidah et al., [9] in 2006 found 23 cases of biphenotypic leukemia in a sample size of 676 in whom only 7 would have met the current accepted guidelines for diagnosing MPAL [9]. Looking at a collection of studies of 7,627 new acute leukemias, 213 cases were classified as biphenotypic. Of these, roughly 119 , or $1.6 \%$, would still be classified as MPAL [6]. The WHO definition represents a refinement of criteria, which is manifested by a much lower reported incidence of the disease.

\section{Cytogenetic and Molecular Features}

Within this newly recognized group of mixed phenotype acute leukemias we find further subcategories based on cytogenetic and molecular features. In general, many patients with MPAL have complex cytogenetics with normal karyotypes seen in only $13 \%$ of patients in one case series [10]. A report of a group of pediatric patients by Manola in 2008 reported that 29 of 33 patients $(88 \%)$ had some form of cytogenetic aberrancies. Overall there exists a broad range in the incidence of cytogenetic abnormalities, ranging from $59 \%-91 \%$ of cases [11], but irrespective of the study, the literature clearly suggests that the vast majority patients had some form of cytogenetic irregularity.

The significance of categorizing chromosomal abnormalities is manifested by the inclusion of two translocations, $t(9 ; 22)$ and $\mathrm{t}(\mathrm{v} ; 11 \mathrm{q} 23)$ in the WHO 2008 criteria [1]. The type and degree of cytogenetic alterations appears to be affected by age. $t(9 ; 22)$, resulting in the Philadelphia chromosome, tends to occur in patients of older age and is in fact, the most common chromosomal change seen in this group [11]. One study by Lee at al., [12] published in 2008 found that roughly $32 \%$ of patients with a biphenotypic diagnosis were found to be Philadelphia chromosome positive [12] while only $3 \%$ were reported to be positive in the pediatric population [8]. A high white count is typical of patients with the $\mathrm{Ph}$ chromosome with the median reported to be $41.8 \times 109 / \mathrm{L}$ [13]. The majority of these patients tend to express a B cell/myeloid combination of cell surface markers. By contrast, younger patients tend to most frequently have chromosome changes involving (v;11q23) causing a form of MLL gene rearrangement $[8,10]$. These patients, too, were noted to have an elevated white count at presentation [11]. In the adult population, only roughly $8 \%$ of patients have been noted to carry the MLL rearrangement [10]. Identifying some of these changes translate directly to therapeutic decision making. The two aforementioned genetic abnormalities do seem to have some varying partiality for age. However, many of the following changes do not necessarily have such strong evidence of correlation with age, gender or immunophenotype [4]. Various abnormalities, including del (1) (p32) and $t(2 ; 5$ p13;p13 15.3) have been described in case reports in the pediatric biphenotypic population [11]. Del 1p32 has also been described in pediatric ALL and tends to carry a favorable prognosis [14]. Monosomy 5 has been described in patients with expression of B cell/T cell and myeloid/T cell leukemias. Monosomy 5 is often seen in conjunction with a complex karyotype and portends a worse prognosis [11]. Monosomy 7 or del (7q) has also been reported. Both of these changes generally portend a poorer prognosis. These changes in chromosome 5 and 7 are also seen in patients with MDS and AML. One cytogenetic change worth mentioning involves del (6q) which, when found, represents a favorable prognosis [15]. There remain a host of other abnormalities, including $\mathrm{t}(10 ; 11)$, trisomy 8 , polysomy $8, t(7 ; 12)$ and $t(8 ; 12)$, all of which have reported in the literature [11].

\section{Treatment}

There are no firm, data-driven established guidelines for the treatment of patients with MPAL. Overall the literature is varied and controversial especially regarding the choice of an induction chemotherapy regimen and the role of Hematopoietic Stem Cell Transplantation (HSCT). In childhood MPAL, there are reports of using modified ALL regimens originally designed for high risk lymphoblastic patients. Many of the drugs in these regimens have activity in myeloid leukemias as well. Once consolidated, patients were maintained on weekly chemotherapy for up to 120 weeks [9].

The St. Jude's Research hospital experience in a pediatric population suggests that ALL-type regimens seem to be more efficacious than AML types. 20 of the 35 patients had a T/Myeloid marker pattern and 12 of 35 had B/Myeloid markers. Two patients had T/Myeloid and B cell markers and 1 patient had an undifferentiated leukemia. No major difference was noted between 
these two phenotypic groups. $83 \%$ of the patients in this study who received an ALL type regimen were able to achieve a complete remission after induction compared with $52 \%$ in the AML group. Additionally, $80 \%$ of patients in the AML group who did not receive a complete remission were able to when re-treated with an ALL regimen [16]. In this pediatric group, many patients were maintained on a 120 week regimen. Overall, a total of $91 \%$ of the 35 patients in the study were able to achieve a CR. $49 \%$ of these patients remained leukemia free survivors. The study did not report data on overall survival as defined by initial induction therapy regimen. Based on their findings, the authors suggest that patients who achieve a molecular remission, defined as $<0.01 \%$ blasts, might not have to move forward with transplant altogether. Patients who had $>1 \%$ blasts were recommended to undergo transplant evaluation [16].

In 2011, Matutes et al., [7] reported on outcomes in a group of a 67 patients, comprised of both adult and pediatric age patients [7]. In the study, 27 patients received an ALL type regimen, 34 received an AML regimen with 2 receiving additional imatinib, 5 received a combination AML/ALL therapy and 1 patient received only imatinib therapy. Twenty moved forward with hematopoietic stem cell transplantation once in remission. $85 \%$ of patients receiving an ALL regimen went into a complete remission versus $41 \%$ of patients receiving an AML regimen. Three of the five patients who received a combination regiment achieved a complete remission. Interestingly, when looking at specific characteristics, median survival was 8 months for Ph positive patients compared with 139 months for patients with normal karyotypes compared with 28 months for patients complex cytogenetics. Median overall survival was noted to be only 11 months for patients treated with an AML regimen versus 139 months for patients who received an ALL treatment course.

A 2008 study by Mikulic et al., [17] reviewed the outcomes of 21 patients defined as biphenotypic acute leukemias based on the EGIL scoring system [17]. In their review, $72 \%$ of patients receiving high dose chemotherapy achieved a CR. At 5 years, overall survival was quoted at $21 \%$. When broken down, by therapy, ALL regimens appeared to be more effective. All patients receiving an ALL regimen were noted to be in a CR when compared with $60 \%$ when treated with an AML regimen. Some authors advocate the use of a combined AML and ALL chemotherapy regimens for remission induction $[18,19]$. In the analysis by Zhang et al., of 40 patients, the rate of first complete remission after chemotherapy was $71.4 \%$ when a combined AML/ALL regimen was used versus $42.9 \%$ when either an ALL or AML regimen was selected [18].

The question of transplantation remains controversial. There is a paucity of data regarding outcomes since the WHO reorganization of these leukemias in 2008. There are no hard and fast strategies to guide the clinician in deciding in whom and when in the treatment course transplantation should occur. Unfortunately, in the evaluation by Matute, there was no report on the outcomes for the 20 patients who underwent either an autologous or allogeneic transplant. In a 3 patient case series reported by Aribi et al., in 2007 [20], 3 patients received allogeneic transplantation after having failed a multitude of therapies. After transplantation all were alive and in remission with a median duration of 4 years [20]. In 2010, Gerr et al., reported a poor prognosis in bilineal acute leukemias and stating that only 2 of their 6 patients were still alive at the time of reporting, both of whom had received an allogeneic transplant [10]. The use of haploidentical HSCT has been employed with some suggestion of efficacy. In their retrospective analysis, Zhang et al., looked at the use of haploidentical hematopoietic stem cell transplantation. Patients who were treated with chemotherapy alone had a one year survival rate of $37.5 \%$ as compared with $60 \%$ in patients treated with chemotherapy followed by haploidentical HSCT [18]. The use of haploidentical transplantation offers advantages to the patient such as shorter wait times for a potential donor. Given the generally poor prognosis of MPAL, coupled with newer sources of hematopoietic stem cells and improved techniques to minimize and manage graft versus host disease, transplant seems to be an important option in the management of this leukemia.

There are limited studies evaluating the appropriate transplant regimens in patients with biphenotypic acute leukemia [21]. A retrospective analysis of 59 patients examined the use of a standard conditioning regimen consisting of Total Body Irradiation (TBI) with Cyclophosphamide (CY) or CY with busulfan. The intensified regimen consisted of $\mathrm{TBI}+\mathrm{CY}+$ etoposide or fludarabine+cytarabine plus $\mathrm{TBI}+\mathrm{CY}+$ etoposide. The 5 year relapse rate for all patients after allogeneic transplantation was $52.9 \%$. $80.8 \%$ of patients in the standard conditioning group had relapsed at 5 years compared with $28.8 \%$ of patients in the intensified conditioning group. Additionally, the overall 5 year survival rate was $23.8 \%$ and $64 \%$ in the standard and intensified groups. Taken together, these data suggest that if the decision is made to move forward with transplantation, for patients who can tolerate, a more aggressive regimen might be in order.

\section{Summary and Conclusions}

Mixed lineage leukemias are a rare entity within the spectrum of acute leukemias. The incidence of these leukemias is likely in the $2 \%$ - 3\% range. The WHO 2008 classification system has led to a simplification of categorizing these conditions. Fewer, but more specific and robust surface markers have been incorporated to help remove ambiguity in diagnosis. Additionally, specific inclusion criteria, such as exclusion of $t(8 ; 21)$ help to ensure that leukemias are not misplaced. Though these have led to a refinement of the diagnosis of MPAL, many questions regarding how to manage these patients remain.

Mixed phenotypic acute leukemias are generally thought to have a generally poor prognosis with overall survival quoted as low as 10 months. Analysis of cytogenetics is becoming increasingly important as potential targets for therapy in the future. In particular the presence of the Philadelphia of chromosome should always be checked by FISH as it is fairly common and would affect the treatment. Patients with complex cytogenetics or $\mathrm{Ph}$ chromosome tend to fare even worse than their other MPAL counterparts. A host of other cytogenetic abnormalities have been discovered, though it is not entirely clear what, if any predictive or prognostic value these may have.

There are no agreed upon treatment protocols and standardization has been difficult to come by. In the absence of prospective randomized data to guide treatment decisions, it has been our practice at Cedars-Sinai Medical Center to use an adult ALL regimen such as hyper-CVAD for young fit patients as it is more inclusive than an AML regimen. A tyrosine kinase inhibitor such as imatinib or dasatinib should be employed in conjunction with cytotoxic chemotherapy for patients with the Philadelphia chromosome. The role of transplantation is not clear. Given the bad prognosis we tend to favor an allogeneic transplantation for all medically fit patients in first remission. Future studies regarding the choice of initial regimen and the role of transplant are clearly necessary even in the era of molecularly targeted therapies. 


\section{References}

1. Chisté M, Vrotsos E, Zamora C, Martinez A (2013) Chronic lymphocytic leukemia/small lymphocytic lymphoma involving the aortic valve. Ann Diagn Pathol 17: 295-297.

2. Catovsky D, Matutes E, Buccheri V, Shetty V, Hanslip J, et al. (1991) A classification of acute leukaemia for the 1990s. Ann Hematol 62: 16-21.

3. Bene MC, Castoldi G, Knapp W, Ludwig WD, Matutes E, et al. (1995) Proposals for the immunological classification of acute leukemias. European Group for the Immunological Characterization of Leukemias (EGIL). Leukemia 9: 1783-1786.

4. Gao C, Sands AM, Sun J (2012) Mixed Phenotype Acute Leukemias. NAJ Med Sci 5: 119-122.

5. Arber DA, Snyder DS, Fine M, Dagis A, Niland J, et al. (2001) Myeloperoxidase immunoreactivity in adult acute lymphoblastic leukemia. Am J Clin Pathol 116: 25-33.

6. Weinberg OK, Arber DA (2010) Mixed-phenotype acute leukemia: historica overview and a new definition. Leukemia 24: 1844-1851.

7. Matutes E, Pickl WF, Van't Veer M, Morilla R, Swansbury J, et al. (2011) Mixed-phenotype acute leukemia: clinical and laboratory features and outcome in 100 patients defined according to the WHO 2008 classification. Blood 117: 3163-3171.

8. Al-Seraihy AS, Owaidah TM, Ayas M, El-Solh H, Al-Mahr M, et al. (2009) Clinical characteristics and outcome of children with biphenotypic acute leukemia. Haematologica 94: 1682-1690.

9. Owaidah TM, Al Beihany A, Iqbal MA, Elkum N, Roberts GT (2006) Cytogenetics, molecular and ultrastructural characteristics of biphenotypic acute leukemia identified by the EGIL scoring system. Leukemia 20: 620-626.

10. Gerr H, Zimmermann M, Schrappe M, Dworzak M, Ludwig WD, et al. (2010) Acute leukaemias of ambiguous lineage in children: characterization, prognosis and therapy recommendations. Br J Haematol 149: 84-92.

11. Manola KN (2013) Cytogenetic abnormalities in acute leukaemia of ambiguous lineage: an overview. Br J Haematol 163: 24-39.
12. Lee JH, Min YH, Chung CW, Kim BK, Yoon HJ, et al. (2008) Prognostic implications of the immunophenotype in biphenotypic acute leukemia. Leuk Lymphoma 49: 700-709.

13. Wang Y, Gu M, Mi Y, Qiu L, Bian S, et al. (2011) Clinical characteristics and outcomes of mixed phenotype acute leukemia with Philadelphia chromosome positive and/or bcr-abl positive in adult. Int J Hematol 94: 552-555.

14. Cave H, Suciu S, Preudhomme C, Poppe B, Robert A, et al. (2004) Clinical significance of HOX11L2 expression linked to $t(5 ; 14)(q 35 ; q 32)$, of HOX11 expression, and of SIL-TAL fusion in childhood T-cell malignancies: results of EORTC studies 58881 and 58951. Blood 103: 442-450.

15. Park JA, Ghim TT, Bae Kw, Im HJ, Jang SS, et al. (2009) Stem cell transplant in the treatment of childhood biphenotypic acute leukemia. Pediatr Blood Cancer 53: 444-452.

16. Rubnitz JE, Onciu M, Pounds S, Shurtleff S, Cao X, et al. (2009) Acute mixed lineage leukemia in children: the experience of St Jude Children's Research Hospital. Blood 113: 5083-5089.

17. Mikulic M, Batinic D, Sucic M, Davidovic-Mrsic S, Dubravcic K, et al. (2008) Biological features and outcome of biphenotypic acute leukemia: a case series. Hematol Oncol Stem Cell Ther 1: 225-230.

18. Zhang C, Zhang X, Chen XH, Gao L, Gao L, et al. (2013) Features and clinical outcomes in 40 patients with mixed-lineage acute leukemia in a single center. Hematology 18: 309-314.

19. Zheng C, Wu J, Liu X, Ding K, Cai X, et al. (2009) What is the optimal treatment for biphenotypic acute leukemia? Haematologica 94: 1778-1780.

20. Aribi A, Bueso-Ramos C, Estey E, Estrov Z, O'Brien S, et al. (2007) Biphenotypic acute leukaemia: a case series. Br J Haematol 138: 213-216.

21. Liu QF, Fan ZP, Wu MQ, Sun J, Wu XL, et al. (2013) Allo-HSCT for acute leukemia of ambiguous lineage in adults: the comparison between standard conditioning and intensified conditioning regimens. Ann Hematol 92: 679-687. 